\title{
Crescimento, produção de óleo essencial e trocas gasosas em orégano influenciados por diferentes lâminas de irrigação
}

\author{
Growth, essential oil production and gas exchange in oregano affected by different irrigation depth
}

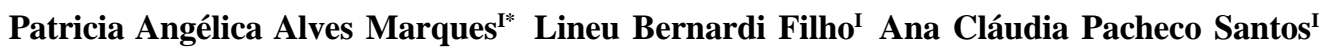

\begin{abstract}
- NOTA -
RESUMO

O orégano é uma planta aromática da família Lamiaceae muito comum na região do mediterrâneo. Ele é rico em óleo essencial e muito usado como tempero na preparação de alimentos. Conhecer o comportamento da espécie em relação às características climáticas da região de plantio é essencial para o sucesso do cultivo. Assim, um experimento foi conduzido com o objetivo de analisar o efeito de cinco lâminas de irrigação em condições de campo, na produção de óleo essencial e nas trocas gasosas da planta de orégano. As lâminas calculadas com base na ECA (Evaporação do Tanque Classe A) foram 0 (sem reposição de água); 25;

analyze the effect of five different depth irrigations under field conditions in the production of essential oil and gas exchange in the oregano plant. The water depths were estimated based on the CPE (Class A pan evaporation): 0 (without water replacement); 25; 50; 75 and 100\% CPE. The experiment was carried out in Presidente Prudente, SP, Brazil, from February to May 2006. A linear positive relation was observed to depths irrigations increment in the fresh matter for aerial plant parts, dry matter for aerial and root zone plant parts; the root/aerial ratio; content and yield of essential oil. The use of 100\% ECA showed the best oregano growth and essential oil production. The different depths irrigation did not affect the plant gas exchange.
\end{abstract} 50; 75 e 100\% ECA. O experimento foi instalado em Presidente Prudente, em São Paulo (SP), no período de fevereiro a maio de 2006. Observou-se uma relação linear positiva com o incremento das lâminas de irrigação para as variáveis matéria fresca da parte aérea, matéria seca da parte aérea e da raiz; relação raiz/parte aérea, teor e rendimento do óleo essencial. O uso da lâmina de 100\% ECA apresentou os melhores resultados de crescimento das plantas de orégano e de produção de óleo essencial. As diferentes lâminas de irrigação não afetaram as trocas gasosas da planta.

Palavras-chave: Origanum vulgare, manejo da irrigação, tanque classe A.

\section{ABSTRACT}

The oregano is an aromatic plant of the Lamiaceae family, very common in the Mediterranean region, rich in essential oil and very used as spice in food preparation. Knowledge about the species behavior with respect to the region climatic characteristics of planting is essential to the cultivation success. Thus, one experiment was carried out to
Key words: Origanum vulgare, irrigation scheduling, class A pan evaporation.

Antes de se iniciar o cultivo de plantas medicinais e aromáticas em escala comercial, faz-se necessário conhecer o comportamento da espécie em relação às características climáticas da região de plantio, aos tratos culturais e aos fatores bióticos que são responsáveis pelo desenvolvimento da planta. De acordo com SILVA et al. (2002), poucos são os trabalhos referentes ao efeito da deficiência hídrica em plantas medicinais, principalmente em relação aos produtos do metabolismo secundário. O efeito varia com o tipo, a intensidade e a duração do estresse, podendo aumentar ou diminuir o teor de óleos essenciais.

'Programa de Pós-graduação em Agronomia, Departamento de Ciências Agrárias, Universidade do Oeste Paulista (UNOESTE). Rod. Raposo Tavares, km 572, 19067-175, Presidente Prudente, SP, Brasil. E-mail: pmarques@unoeste.br. *Autor para correspondência. 
O lento desenvolvimento de deficiência hídrica pode induzir o ajustamento osmótico em algumas espécies, resultando na manutenção da turgescência celular a baixos potenciais hídricos. Este é um importante mecanismo de tolerância à seca, porque capacita a planta a continuar com o seu crescimento normal e com a sua produção rentável, por meio da realização de ajustes estomáticos e fotossintéticos (KUMAR \& SINGH, 1998). Desse modo, o objetivo deste trabalho foi analisar o efeito de cinco lâminas de irrigação na produção do óleo essencial e nas trocas gasosas do orégano (Origanum vulgare L.).

O experimento foi realizado no período de 24 de fevereiro a 24 de maio de 2006, em Presidente Prudente, São Paulo (SP), latitude $22^{\circ} 07^{\prime} 04^{\prime \prime}$ S e longitude $51^{\circ} 22^{\prime} 05^{\prime}$ ' W, altitude de $435,5 \mathrm{~m}$. O clima é definido como Aw pela classificação de Köppen, mesotérmico com verões quentes e invernos secos. O solo é classificado como Argissolo Vermelho-Amarelo, Eutróficos de textura arenosa/média (EMBRAPA, 1999). As características químicas do solo foram determinadas, para a camada de $0-20 \mathrm{~cm}$, segundo RAIJ et al. (2001), sendo: $\mathrm{P}$ (resina), 24mg dm${ }^{-3}$; M.O., $5 \mathrm{mg} \mathrm{dm}^{-3}$; $\mathrm{pH}$ $\left(\mathrm{CaCl}_{2}\right), 6,3 ; \mathrm{K}^{+}, 2,2 \mathrm{mmolc} \mathrm{dm}{ }^{-3} ; \mathrm{Ca}^{++}, 19 \mathrm{mmolc} \mathrm{dm}^{-3}$; $\mathrm{Mg}^{++}, 14 \mathrm{mmol} \mathrm{dm}^{-3}$ e $\mathrm{Al}^{+3}, 0 \mathrm{mmol} \mathrm{dm}^{-3}$; V (saturação por bases), 69\%; M (saturação por alumínio), 0\%. O solo do experimento não recebeu adubação nem calagem por tratar-se de área comumente utilizada para plantio de olerícolas com valor de V próximo a 70\% e teores de nutrientes adequados (RAIJ et al., 1996).

As mudas de orégano (Origanum vulgare L.) foram obtidas por estacas plantadas em bandejas de polipropileno de 120 células e mantidas em viveiro por 30 dias. O espaçamento utilizado foi de $0,6 \mathrm{~m}$ x 0,3m. Durante o ciclo de desenvolvimento os tratos culturais realizados constaram de capina manual e controle de infestação de formigas utilizando iscas. O delineamento utilizado foi o inteiramente casualizado, constando de cinco tratamentos de lâminas de irrigação estimadas com base nas leituras realizadas diariamente no tanque classe A(ECA) da estação meteorológica da UNOESTE, em Presidente Prudente, SP, sendo: T1 - sem reposição de água - 0, T2 - 25, T3-50, T4 - 75 e T5-100\% ECA, com cinco repetições. Os canteiros foram erguidos manualmente, resultando em 25 parcelas de $2,8 \mathrm{~m}^{2}$, que foram separadas por uma barreira física a fim de impedir a contaminação da umidade das parcelas. Foi utilizado um irrigador manual com calibrações a cada $500 \mathrm{~mL}$. A água foi distribuída de maneira uniforme diariamente ao final da tarde. Aos 45 e aos 90 dias após o plantio (DAP), foram realizadas as medições de taxa de assimilação de $\mathrm{CO}_{2}(\mathrm{~A})$, condutância estomática (gs), transpiração (E) e concentração interna de $\mathrm{CO}_{2}(\mathrm{Ci})$. As medidas foram tomadas entre 10 e 12 horas (quatro folhas por tratamento), utilizando-se um analisador portátil a gás infravermelho (modelo CIRAS 2PPSystem). O potencial hídrico foliar ( $\psi$ h) foi medido às 7 horas por meio de uma Câmara de Scholander. Utilizou-se o balanço hídrico semanal conforme PEREIRA et al. (2002), considerando-se o armazenamento de água no solo (CAD) de 100mm.

Aos 90DAP, as plantas foram coletadas pela manhã, em torno das 9 horas, quando as plantas possuem mais óleo essencial (LOPES et al., 2001). As folhas frescas foram pesadas em balança analítica, obtendo-se a matéria fresca da parte aérea (MFPA). Amostras de 100g de MFPA foram utilizadas para a extração do óleo essencial por meio da hidrodestilação em aparelho de Clevenger modificado. O teor de óleo (Teor) foi obtido diretamente como percentagem da MFPA. Já o rendimento de óleo em gramas por planta (Rend) foi calculado por meio da concentração multiplicada pelo valor de MFPA. As folhas e raízes foram secas em estufa a $45^{\circ} \mathrm{C}$, até atingirem peso constante. Utilizando uma balança analítica, foram determinadas a matéria seca das folhas (MSPA) e a matéria seca das raízes (MSR). Todas as variáveis foram submetidas à análise de variância, e os tratamentos foram comparados pelo teste de Scott-Knott (5\% de significância). Quando houve diferença significativa entre os tratamentos, os dados referentes a todas as repetições foram submetidos à análise de regressão pelo programa estatístico SISVAR 4.6.

No período estudado, observou-se a ocorrência de $96 \mathrm{~mm}$ de precipitação pluvial. O Balanço hídrico semanal (Figura 1) demonstra o excedente hídrico no início, seguido de déficit hídrico até o final do ciclo de desenvolvimento do orégano. A lâmina total de irrigação aplicada para 0; 25; 50; 75 e 100\% ECA foi de $0 ;$ 54; 108,41; 162,62 e 216,82mm, respectivamente.

Pelos parâmetros de produção (Figura 2), nota-se que as variáveis relacionadas ao crescimento e à produção, ou seja, MFPA, MSPA, MSR, relação Raiz/PA, teor e rendimento de óleo foram influenciadas pelas lâminas de irrigação com respostas lineares, resultados semelhantes aos obtidos por SILVA et al. (2002) em Melaleuca alternifolia. O tratamento com 100\% ECA apresentou a maior produção de MFPA e MSPA, equivalente a um acréscimo de 158\% e 181\%, respectivamente, em relação ao tratamento sem irrigação. Para o teor de óleo essencial, os melhores resultados foram obtidos com os tratamentos irrigados com 50\%, 75\% e 100\% ECA. Para a camomila, PIZARD et al. (2006) também observaram maior teor e rendimento de óleo com o incremento da lâmina de irrigação. Dessa maneira, pode-se concluir que o orégano é uma planta 


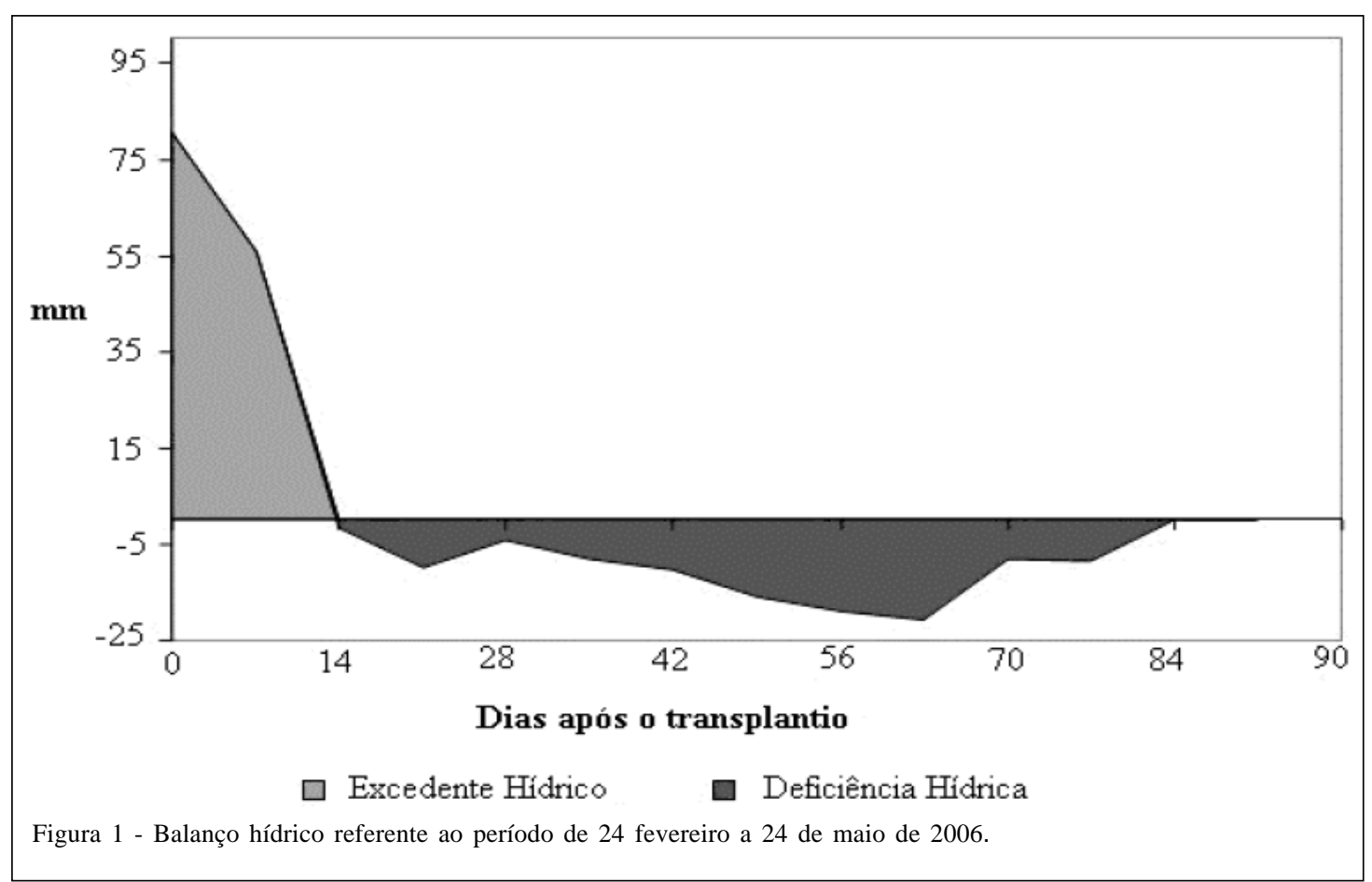

que, em ambientes sem déficit hídrico, tem a produção de princípios ativos favorecidos, como discutido em ANDRADE \& CASALI (1999).

O rendimento de óleo essencial é função de seu teor no tecido e da produção de material vegetal (OLIVEIRA JÚNIOR et al., 2005). Como os tratamentos influenciaram significativamente a MFPA, o rendimento também apresentou resposta linear positiva, sendo o maior valor $\left(0,32 \mathrm{~g}\right.$ planta $\left.^{-1}\right)$ obtido para o tratamento $100 \%$ ECA e o menor $\left(0,096\right.$ glanta $\left.^{-1}\right)$ para o tratamento sem irrigação ( $0 \%$ ECA). Resultados semelhantes foram encontrados por MARCUM \& HANSON (2006), em que lâminas de irrigação inferiores à evapotranspiração provocaram reduções no rendimento de óleo essencial da hortelã. Esses resultados corroboram a opinião de ANDRADE \& CASALI (1999), os quais consideram a água essencial à vida e ao metabolismo das plantas, esperando-se, portanto, que a produção de princípios ativos seja maior em ambientes sem déficit hídrico. Observou-se que, tanto o crescimento radicular, quanto o crescimento da parte aérea de plantas de orégano, foram afetados positivamente com o aumento da lâmina de irrigação. Para todos os parâmetros estudados, o uso da lâmina de irrigação de 100\% ECA proporcionou os maiores índices de crescimento em plantas de orégano.

A deficiência hídrica afeta processos fisiológicos e metabólicos da planta, incluindo o crescimento celular, a síntese de proteínas, a abertura estomática e o transporte de nutrientes (NEPOMUCENO et al., 1998). Na tabela 1, são apresentados os valores de trocas gasosas das plantas de orégano submetidas a diferentes intensidades de deficiência hídrica, medidos aos 45 e 90DAP. Aos 45DAP, observou-se que, em relação aos parâmetros transpiração (E), condutância estomática (gs), concentração intracelular de $\mathrm{CO}_{2}(\mathrm{Ci})$ e potencial de água foliar $(\psi h)$, não houve diferenças entre os tratamentos. Já em relação à fotossíntese líquida (A), observou-se que as plantas submetidas ao tratamento sem irrigação (0\%ECA) apresentaram valores reduzidos em relação aos tratamentos irrigados. Entretanto, com o aumento da intensidade do estresse, houve uma tendência de diminuição da atividade fotossintética das plantas. Já aos 90DAP não houve diferença significativa para todas as variáveis analisadas.

De acordo com CORNIC (2000), algumas das mudanças metabólicas que ocorrem como resultado da deficiência hídrica são uma consequência da resistência do aparato fotossintético à desidratação. CHAVES et al. (2002) afirmam que plantas herbáceas de clima mediterrâneo apresentam relativa tolerância à desidratação de seus tecidos, levando a uma considerável resistência do aparato fotossintético à condição de seca.

O ajustamento osmótico é um importante mecanismo de tolerância à seca, porque capacita a

Ciência Rural, v.39, n.6, set, 2009. 

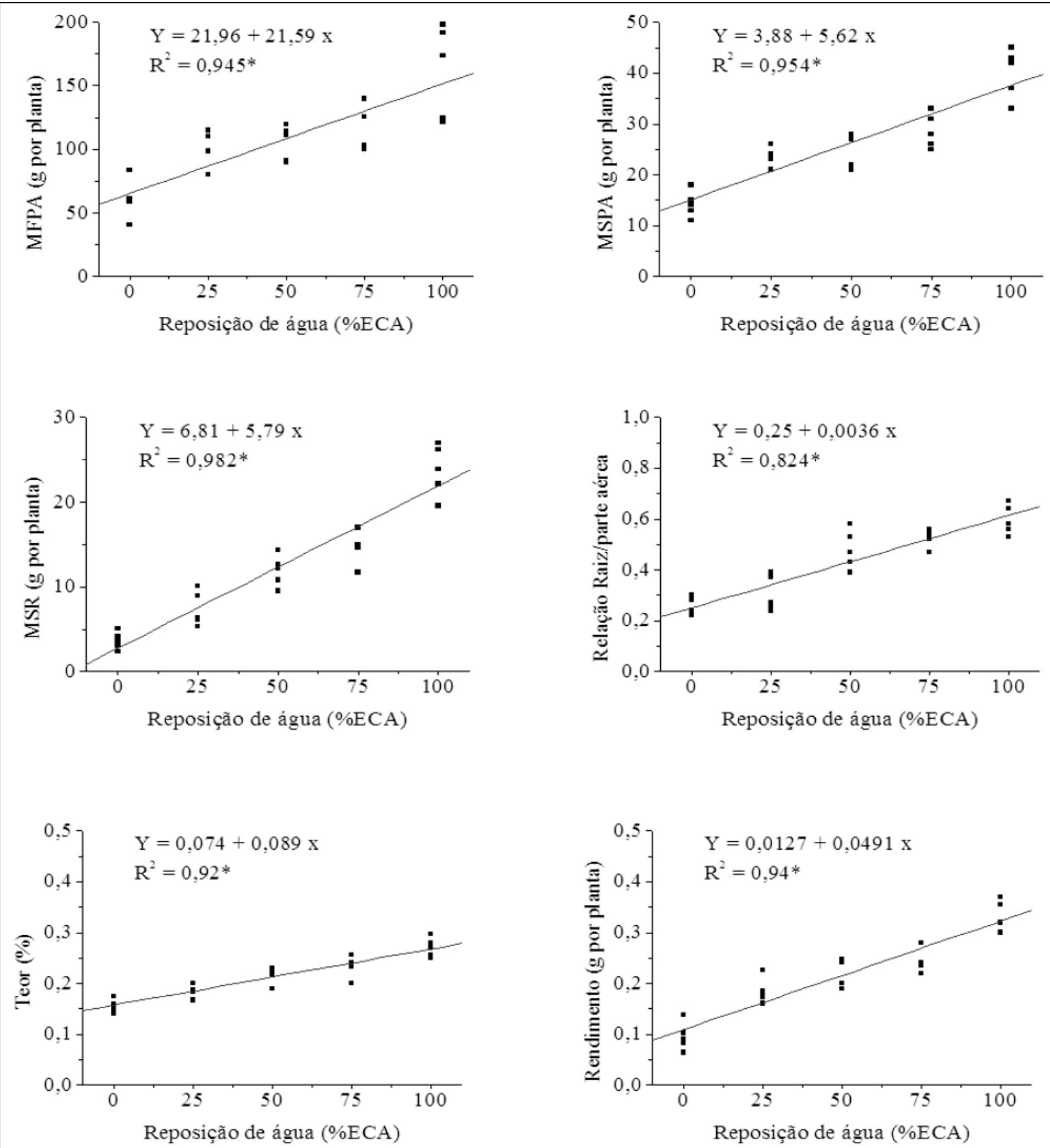

Figura 2 - Resultados de matéria fresca da parte aérea (MFPA), matéria seca da parte aérea (MSPA), matéria seca de raiz (MSR), relação Raiz/parte aérea, teor de óleo essencial (Teor) e rendimento de óleo essencial (Rend) em função da lâmina de irrigação.

planta a continuar com o seu crescimento normal e com sua produção rentável, por meio da realização de ajustes estomáticos e fotossintéticos (KUMAR \& SINGH, 1998). Os resultados indicam que o orégano é uma espécie que apresenta a capacidade de manter seus mecanismos fisiológicos mesmo sob condições de deficiência hídrica. Dessa maneira, o investimento da planta no ajuste osmótico pode ter ocasionado a diminuição no seu crescimento. Concluiu-se então que o uso da lâmina de 100\% ECA apresentou os melhores resultados de crescimento das plantas de orégano e de produção de óleo essencial. As diferentes lâminas de irrigação não afetaram as trocas gasosas nas plantas de orégano.

Ciência Rural, v.39, n.6, set, 2009. 
Tabela 1 - Valores de transpiração (E), condutância estomática (GS), concentração intracelular de $\mathrm{CO}_{2}$ (Ci), potencial de água foliar ( $\psi$ h) e fotossíntese líquida (A) aos 45 e aos 90 dias após o plantio (DAP).

\begin{tabular}{|c|c|c|c|c|c|c|c|c|c|c|}
\hline \multirow{2}{*}{ Tratamento } & \multicolumn{5}{|c|}{ 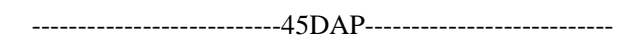 } & \multicolumn{5}{|c|}{ 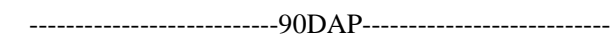 } \\
\hline & $\mathrm{E}$ & GS & $\mathrm{Ci}$ & $\psi h$ & A & $\mathrm{E}$ & GS & $\mathrm{Ci}$ & $\psi h$ & A \\
\hline $0 \%$ ECA & 3,20a 1 & $146,60 \mathrm{a}$ & $258,40 \mathrm{a}$ & 1,00a & $6,20 \mathrm{~b}$ & $3,45 a$ & $171,00 \mathrm{a}$ & $250,25 a$ & $0,75 a$ & $7,50 \mathrm{a}$ \\
\hline $25 \%$ ECA & $3,40 \mathrm{a}$ & $201,80 a$ & $273,60 a$ & $0,92 \mathrm{a}$ & $7,20 \mathrm{a}$ & $3,45 a$ & $217,25 a$ & $266,75 a$ & $0,82 \mathrm{a}$ & $8,20 \mathrm{a}$ \\
\hline $50 \%$ ECA & $3,00 \mathrm{a}$ & $175,80 a$ & $216,60 a$ & $1,06 \mathrm{a}$ & $7,80 \mathrm{a}$ & $3,45 a$ & $209,00 a$ & $247,25 a$ & $0,78^{\mathrm{a}}$ & $7,85 a$ \\
\hline $75 \%$ ECA & $4,20 \mathrm{a}$ & $266,40 a$ & $270,40 a$ & $0,98 a$ & $8,60 a$ & $4,22 \mathrm{a}$ & $258,25 a$ & $272,75 a$ & $0,78 a$ & $7,77 a$ \\
\hline $100 \%$ ECA & $3,80 a$ & $247,20 a$ & $257,00 \mathrm{a}$ & $0,97 a$ & $9,80 a$ & 3,93a & $260,75 a$ & $252,25 a$ & $1,04 a$ & $10,42 a$ \\
\hline
\end{tabular}

${ }^{1}$ Médias seguidas de mesma letra, nas colunas, não diferem estatisticamente entre si pelo Teste de Scott-Knott, a 5\% de probabilidade.

\section{REFERÊNCIA}

ANDRADE, F.M.C.; CASALI, V.W.D. Plantas medicinais e aromáticas: relação com 0 ambiente, colheita e metabolismo secundário. Viçosa: Universidade Federal de Viçosa, Departamento de Fitotecnia, 1999. 139p.

CHAVES, M.M. et al. How plants cope with water stress in the field? Photosynthesis and Growth. Annals of Botany, Oxford, v.89, p.907-916, 2002. Disponível em: <http:// aob.oxfordjournals.org/cgi/reprint/89/7/907>. Acesso em: 19 fev. 2009. Doi: 10.1093/aob/mcf105.

CORNIC, G. Drought stress inhibits photosynthesis by decreasing stomatal aperture - not by affecting ATP synthesis. Trends in Plant Sciences, Oakland, n.5, p.187-188, 2000. Disponível em: <http://dx.doi.org/10.1016/S1360-1385(00)01625-3>. Acesso em: 19 fev. 2009. Doi: 10.1016/S13601385(00)01625-3.

EMBRAPA Sistema brasileiro de classificação de solos. Brasília: EMBRAPA-SPI/CNPS, 1999. 412p.

LOPES, R.C. et al. Influência de três regimes hídricos na produção de óleo essencial em sete acessos de Polygonum punctatum Ell. Revista Brasileira de Plantas Medicinais, Botucatu, v.3, n.2, p.7-10, 2001.

KUMAR, A.; SINGH, D.P. Use of physiological indices as a screening technique for drought to tolerance in oilseed Brassica species. Annals of Botany, Oxford, v.81, p.413-420, 1998.

MARCUM, D.B.; HANSON, B.R. Effect of irrigation and harvest timing on peppermint oil yield in California. Agricultural Water Management, Amsterdam, v.82, n.12, p.118-128, 2006. Disponível em: <http://dx.doi.org/ 10.1016/j.agwat.2005.07.022>. Acesso em: 19 fev. 2009. Doi: 10.1016/j.agwat.2005.07.
MASTRO, G. de. et al. Agronomic and technological assessment of oregano (Origanum vulgare ssp.) Biotypes. Acta Horticulturae, Leuven - Bélgica, v.629, p.355-363, 2004.

NEPOMUCENO, A.L. et al. Physiological responses of cotton leaves and roots to water deficit induced by polyethylene glycol. Environmental and Experimental Botany, Amsterdan, v.40, p.29-41, 1998. Disponível em: <http://dx.doi.org/10.1016/ S0098-8472(98)00018-5>. Acesso em: 19 fev. 2009. Doi: 10.1016/S0098-8472(98)00018-5.

OLIVEIRA JÚNIOR, A.C. et al. Teor e rendimento de óleo essencial no peso fresco de arnica, em função de calagem e adubação. Horticultura Brasileira, Brasília, v.23, n.3, p.735739, 2005. Disponível em: <http://www.scielo.br/ scielo.php? script=sci_art text \& pid=S 0102 05362005000300009>. Acesso em: $19 \mathrm{fev}$. 2009. Doi: 10.1590/S0102-05362005000300009.

PEREIRA, A.R. et al. Agrometeorologia: fundamentos e aplicações práticas. Guaíba: Agropecuária, 2002. 478p.

PIZARD, A. et al. Essential oil content and composition of german chamomile (Matricaria chamomilla L.) at diffent irrigation regimes. Journal of Agronomy, Berlim, v.5, n.3, p.451-455, 2006.

RAIJ, B. Van et al. Recomendações de adubação e calagem para o Estado de São Paulo. 2.ed. Campinas: Instituto Agronômico \& Fundação IAC, 1996. 285p. (Boletim técnico, 100).

RAIJ, B. Van et al. Análise química para avaliação de fertilidade de solos tropicais. Campinas: Instituto agronômico, 2001. 285p.

SILVA, S.R.S. et al. Efeito do estresse hídrico sobre características de crescimento e a produção de óleo essencial de Melaleuca alternifolia Cheel. Acta Scientiarum, Maringá, v.24, n.5, p.1363-1368, 2002. 